AlH v non-AIH CLD

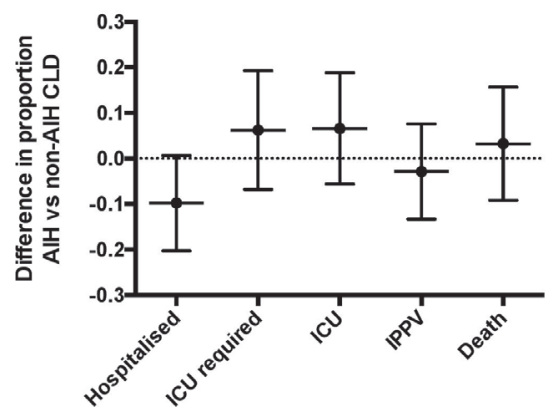

AIH v non-CLD

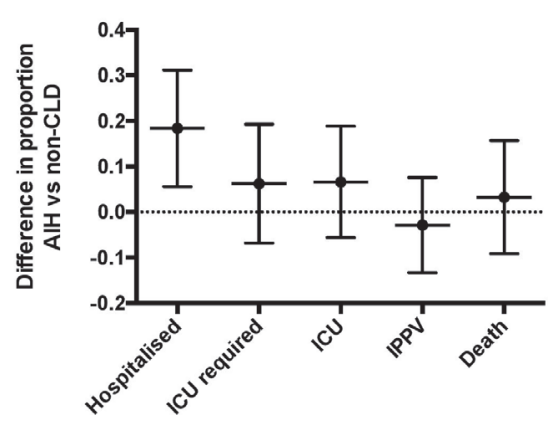

Abstract OTH-1 Figure 1 (A) AlH v non-AlH CLD (B) AlH v non-CLD

eight $(83 \%)$ of $\mathrm{AIH}$ patients were taking one or more immunosuppressive drug. There were no differences in rates of major outcomes between AIH and non-AIH CLD including hospitalisation $(76 \%$ vs $85 \% ; \mathrm{p}=0.06)$, ICU admission $(29 \%$ vs. $23 \%$; $\mathrm{p}=0.240)$, and death $(23 \%$ vs. $20 \%$; $\mathrm{p}=0.643)$. Factors associated with death within the AIH cohort included age (OR 2.16/10 years; 1.07-3.81), Child-Turcotte-Pugh (CTP) class B (OR 42.48; 4.40-409.53), and CTP-C cirrhosis (OR $69.30 ; 2.83-1694.50)$, but not use of immunosuppression. Propensity score matched (PSM) analysis comparing AIH with non-AIH CLD demonstrated no increased risk adverse outcomes including death (+3.2\%; -9.2\%-15.7\%) (Figure 1A). PSM analysis of AIH versus non-CLD patients $(n=769)$ demonstrated increased risk of hospitalisation with $\mathrm{AIH}(+18.4 \%$; 5.6-31.2\%), but equivalent risk of all other outcomes including death (+3.2\%; -9.1\%-15.6\%) (Figure. 1B).

Conclusion Despite immunosuppressive treatment, AIH patients were not at increased risk of adverse outcomes from COVID19 compared to other causes of CLD and to matched cases without liver disease.

\section{OTH-7 INTEGRATED CARE PLANS FOR ALCOHOL-RELATED HIGH IMPACT SERVICE USERS REDUCES HOSPITAL ATTENDANCES AND ADMISSIONS}

Steven Masson*, Stephen Forrest, Laura Jopson, Steven Masson. Liver Unit, Newcastle Hospitals NHS Foundation Trust, Newcastle, UK

\subsection{6/gutjnl-2021-BSG.14}

Introduction Alcohol-related hospital admissions have doubled in the last decade to $>1.3 \mathrm{~m}$ per year in England. Alcoholrelated high impact users are a subgroup who have multiple hospital admissions or attendances related to alcohol during a short time-period. We aimed to determine the effectiveness of integrated care plans in improving outcomes of high intensity users and reducing resource use within our acute hospitals trust (NUTH).

Methods The multidisciplinary trustwide alcohol care team (ACT) agreed case management strategies for a cohort with the highest rates of alcohol-related admissions and/or ED attendences to NUTH. A comprehensive individualised care plan, drawing on several sources (acute hospital, community and addicitions services) was agreed for each. This included details of keyworker contact details, a defined management plan, including threshold for admission, along with recommendations for involvement of safeguarding or statutory services, as apporpriate. This care plan was then made available to staff in ED and Acute admissions, at the point of triage, along with members of the ACT. Care plans, and the cohort of high impact users, were reviewed each six months.

Results Over 2 years, care plans were defined for 29 high impact users (15 male) with a median age 42 (36-84). In the 12 months prior to care plans, they had total 342 admissions to NUTH (mean 12 each) and an additional 521 ED attendances (mean 15 each). In the 12 months after introduction of care plans, they had 220 admissions to NUTH (mean 6 each) and a further $268 \mathrm{ED}$ attendances (mean 8 each). Of this cohort, 9 (31\%) died, mainly of alcohol-related causes, at median 12 (8-29) months after care plan implementation.

Conclusions The introduction of care plans for alcohol-related high impact has resulted in 36\% less hospital admissions and 49\% less ED attendances, within 12 months of their implementation. Despite strategic individualised care coordination, these high impact users continue to have a high risk of subsequent alcohol-related mortality. They require additional attention and ongoing specialist input.

\section{OWE-3 TIMING OF PRE-EMPTIVE TIPS DOES NOT ALTER PATIENT OUTCOME, A UK MULTICENTRE STUDY}

${ }^{1}$ Philip Dunne*, ${ }^{2}$ Jemima Finkel, ${ }^{3}$ Faisal Khan, ${ }^{1}$ Neil Lachlan, ${ }^{3}$ Dhiraj Tripathi, ${ }^{2}$ David Patch, ${ }^{1}$ Adrian Stanley, ${ }^{4}$ Peter Hayes. 'Glasgow Royal Infirmary, Glasgow, UK; ${ }^{2}$ Royal Free Hospital, London, UK; ${ }^{3}$ Queen Elizabeth Hospital, Birmingham, UK; ${ }^{4}$ Royal Infirmary Edinburgh, Edinburgh, United Kingdon

\subsection{6/gutjnl-2021-BSG.15}

Introduction International guidelines suggest consideration of pre-emptive transjugular intrahepatic portosystemic shunt (pTIPS) within $72 \mathrm{~h}$ of endoscopic haemostasis following acute oesophageal variceal bleeding (OVB) in 'high-risk' patients. However, the $72 \mathrm{~h}$ timeframe is arbitrary and pTIPS placement beyond $72 \mathrm{~h}$ has never been explored. Large observational multicentre studies underscore continued equipoise regarding the value of pTIPS and, with the inclusion of a recent randomised control trial (RCT), highlight the difficulty in accessing timely interventional radiology to provide a non-emergency service in real-world clinical practice.

Methods Patients referred to 4 UK tertiary centres for pTIPS between $01 / 01 / 2010$ - 31/12/2018 were included in the prospective data collection. The time to pTIPS from index endoscopy was recorded and pre-defined clinically relevant outcomes were observed relative to two groups, 'early-TIPS' $(<72 \mathrm{~h})$ and 'late-TIPS' (72h - 30-days). Primary outcome was transplant free survival at 1-year. All patients were followedup until 31/12/2020. 


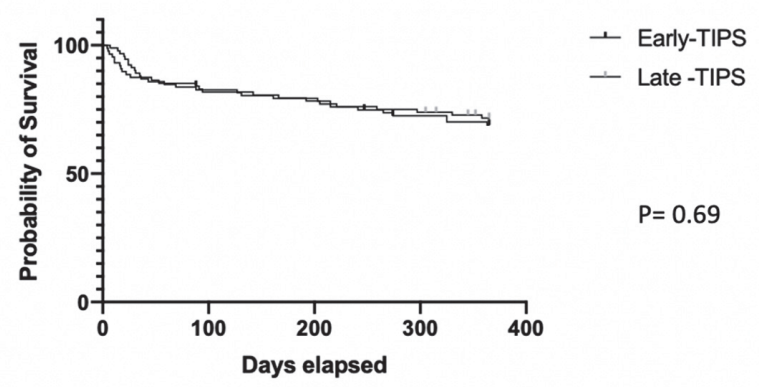

Abstract OWE-3 Figure 1 Survival Analysis - Early vs Late TIPS

Results 180 patients were referred for pTIPS, across the 4 sites. 88 received 'early-TIPS' and 92 received 'late-TIPS'. Propensity score matching determined the early and late pTIPS groups were well matched with no difference in mean age (53 vs 52), predominant aetiology (alcohol liver disease; 61\% vs 67\%) mean MELD (16.42 vs 15.52), mean Child-Pugh Score (9.05 vs 8.9), mean portal pressure gradient (PPG) pre-TIPS (20.06 $\mathrm{mmHg}$ vs $21.58 \mathrm{mmHg}$ ) and mean PPG fall post-TIPS (14.67 $\mathrm{mmHg}$ vs $12.41 \mathrm{mmHg}$ ), respectively. There was no difference in patient outcomes between early and late pTIPS groups respectively; 1-year transplant free survival rate $(69.3 \%$ vs $70.7 \% ; \mathrm{p}=0.69$ ) (Figure 1$)$, 1-year variceal rebleeding rates $(6.8 \%$ vs $11.9 \% ; \mathrm{p}=0.27)$ and cause of death (liver failure; $27.3 \%$ vs $25 \%$; $=0.73$ ).

Conclusion Our study, which is the largest of pTIPS data to date, confirms placement of pTIPS within $72 \mathrm{~h}$ offers no benefit to patient outcome over pTIPS placed between $72 \mathrm{~h}-30$ days. Despite ongoing uncertainty as to whether pTIPS offers survival benefit over modern standards of care (i.e., band ligation and non-selective beta blockers), our results suggest pTIPS could become readily available given the potential easing of time constraints, however our findings should be considered in future RCTs.

\section{OWE-4 NATIONAL AUDIT OF DIAGNOSIS, MANAGEMENT AND SURVEILLANCE IN PRIMARY SCLEROSING CHOLANGITIS IN THE UNITED KINGDOM}

${ }^{1}$ Evangelia Fatourou*, ${ }^{2}$ Sarah Hyde, ${ }^{3}$ Sarah Hyde, ${ }^{4}$ Martine Walmsley, ${ }^{1}$ Graeme Alexander, ${ }^{2}$ Simon Rushbrook, ${ }^{3}$ Palak Trivedi, ${ }^{1}$ Douglas Thorburn, Contributing Investigators UK PSC. 'Sheila Sherlock Liver Centre and UCL Institute for Liver and Digestive Health, Royal Free Hospital, London, UK; ' Department of Hepatology, Norwich Medical School, University of East Anglia, Norwich, UK; ${ }^{3}$ University Hospitals Birmingham, Birmingham, UK; ${ }^{4}$ PSC Support, Oxford, UK

\subsection{6/gutjnl-2021-BSG. 16}

Introduction Primary sclerosing cholangitis (PSC) is a rare disorder and as such clinical care can be heterogeneous. We audited PSC management across the UK against audit standards set by the British Society of Gastroenterology (BSG).

Method All UK PSC investigators were invited to complete an electronic questionnaire on the PSC patient cohort encompassing demographics, diagnosis, bowel and biliary tract cancer surveillance, and risk stratification data (March 2019 - Jan 2021).

Results 1,795 patients across 30 centres (liver units $\mathrm{n}=1548$, general gastroenterology units $\mathrm{n}=247$ ) were included. Median age at diagnosis was 51 years and $56.4 \%$ were men. Magnetic resonance cholangiography (MRCP) was performed as a diagnostic investigation in 1616 patients (90.0\%) and 777 (43.3\%) had a liver biopsy. Most were monitored by a hepatologist $(\mathrm{n}=1610,89.7 \%) .931$ patients $(51.9 \%)$ received nonlicensed therapy with Ursodeoxycholic acid.

785 patients $(43.7 \%)$ had not undergone disease staging or risk stratification within the last 2 years; where performed, it was most commonly by transient elastography ( $\mathrm{n}=645$, $78.7 \%)$. Surveillance for biliary tract cancer was not undertaken in 515 patients $(28.7 \%)$; when performed, it was most commonly by ultrasound (US) $(\mathrm{n}=568,47.1 \%)$ or alternating MRCP/US ( $\mathrm{n}=429, \mathrm{n}=35.6 \%$ ). Ca 19 - 9 was utilised in 730 patients.

Concurrent IBD was present in 1264 patients (70.4\%) with $256(20.3 \%)$ having had a colectomy. Where classified, pancolitis (Montreal classification E3) was the commonest disease distribution $(673 / 939,71.7 \%)$ with $1.6 \%(n=15)$ having isolated ileal disease. In those without IBD, 142 (28\%) patients had not had a colonoscopy and biopsies to exclude diagnosis.

Among those with colitis without previous colectomy $(n=743), 580(78.1 \%)$ underwent annual colonoscopic surveillance; 30 (5.2\%) with dye spray, 230 (39.7\%) with biopsies and dye spray, and $252(43.4 \%)$ with protocol biopsies alone.

Conclusion There is unwarranted variation in the care of patients with PSC in the UK. In particular relating to risk stratification, exclusion of colitis and surveillance for biliary tract and colonic cancer. The lack of uniformity in clinical practice highlights the need for better education of clinicians about PSC management and the potential role of clinical networks for rare liver diseases within the UK.

\section{OWE-5 CARVEDILOL VS. ENDOSCOPIC BAND LIGATION FOR VARICEAL BLEEDING SECONDARY PROPHYLAXIS; LONG- TERM RCT FOLLOW-UP}

${ }^{1}$ Philip Dunne*, ${ }^{2}$ Peter Hayes, ${ }^{3}$ Dhiraj Tripathi, ${ }^{4}$ Joanna Leithead, 'Lyn Smith, 'Dan Gaya, ${ }^{1}$ Ewan Forrest, ${ }^{1}$ Adrian Stanley. ${ }^{1}$ Glasgow Royal Infirmary, Glasgow, UK; ${ }^{2}$ Royal Infirmary of Edinburgh, Edinburgh, UK; ${ }^{3}$ Queen Elizabeth Hospital, Birmingham, UK; ${ }^{4}$ Forth Valley Royal Hospital, Sterling, UK

\subsection{6/gutjnl-2021-BSG.17}

Introduction Carvedilol reduces rates of variceal bleeding and rebleeding by lowering portal pressure. However, an associated pleotropic survival benefit has been proposed. We aim to investigate further by undertaking long-term follow-up of a multicentred randomised control trial.

Methods The index study randomised 64 cirrhotic patients with clinically confirmed acute oesophageal variceal bleeding between June 2006 and December 2011 to receive either carvedilol or endoscopic band ligation (EBL). ${ }^{1}$ We reviewed electronic patient records for all patients up to 31/04/2020 and updated the previously defined clinically relevant outcomes.

Results Of those randomised, 26 out of 33 participants received carvedilol in the follow-up period and 28 out of 31 attended for regular EBL sessions. There were no significant differences in baseline characteristics between groups. Mean follow-up for all participants was 2217 days. The mean duration of carvedilol administration was 1267 days. On intention to treat analysis, there was a trend towards improved survival in the carvedilol group, but this did not reach statistical significance $(p=0.09)$. However, on per-protocol analysis, carvedilol administration was significantly associated with improved longterm survival $(\mathrm{p}<0.01)$, as well as fewer liver related deaths $(4 \%$ vs $29 \%, \quad \mathrm{p}=0.02, \mathrm{OR}=0.1)$ and fewer participants 